\title{
Base pair dynamic assisted charge transport in DNA
}

\author{
E. I. Kats ${ }^{1,2}$ and V. V. Lebedev ${ }^{1,3}$ \\ ${ }^{1}$ L. D. Landau Institute for Theoretical Physics, RAS \\ 117940, Kosygina 2, Moscow, Russia; \\ 2 Institut Laue-Langevin, 6 rue Jules Horowitz, BP 156, Grenoble, France; \\ 3 Department of Physics, Weizmann Institute of Science, 76100, Rehovot, Israel.
}

(October 26, 2018)

\begin{abstract}
An $1 d$ model with time-dependent random hopping is proposed to describe charge transport in DNA. It admits to investigate both diffusion of electrons and their tunneling between different sites in DNA. The tunneling appears to be strongly temperature-dependent. Observations of a strong (exponential) as well as a weak distance dependence of the charge transfer in DNA can be explained in the framework of our model.
\end{abstract}

PACS numbers: $87.15-\mathrm{v}, 87.14 . \mathrm{Gg}$

Electronic transport is a ground to a wide range of important biological processes in DNA. Besides, the phenomenon has a fundamental physical interest, since the transport properties of biomolecules are expected to differ considerably from those of macroscopic conductors. And at last, very recently material scientists also turned their attention to charge migration in DNA for the development of DNA-based molecular technologies.

Although first attempts to measure DNA conductivity [1], and to give a theory of the phenomenon [2] have been made almost 40 years ago, the question concerning charge transport through DNA remains unsettled, and there is an impressive quantity of unexplained or partially explained data. Different publications report frequently contradictory results. Two kinds of techniques for getting information on charge transport in DNA are used. First, direct or indirect electrical conductivity measurements on micrometer-long DNA ropes are performed [3]. Experimental results obtained in this technique are ambiguous. DNA conductivity $\sigma$ was reported as almost metallic of the order $10^{4} \Omega^{-1} \mathrm{~cm}^{-1}$ [3] (in a recent publication the authors claim they observed even proximity induced superconductivity in DNA) or semiconducting with $\sigma \simeq 0.1 \Omega^{-1} \mathrm{~cm}^{-1}[6]$. Very recently experimental techniques have progressed to the point where the conductivity on individual 10 $\mathrm{nm}$ long double stranded molecules was measured [7] and the result implies that DNA is a good insulator. Clearly this frustrating situation with conductivity measurements means that there are many relevant factors which can influence the charge transport in DNA by different way and which are hardly controlled in real experiments. The second technique, related to fluorescence quenching measurements on DNA strands, doped with donor and acceptor molecules [8, 23], seems more reliable, and it is our main concern here. In this technique photo-excitation of a donor associated with the stack of base pairs in some fashion, allows transfer of an electron to the stack. The migrating electron is trapped finally at the acceptor site, and charge transfer is monitoring by the yield of a chemical reaction accompanying the trapping process. The transfer rate is usually assumed to be characterized by a simple exponential law $\exp (-\beta x)$, where $x$ is the donor-acceptor separation. Fitting to this law gives values of $\beta$ ranging from $0.1 \AA^{-1}$ to $1.4 \AA^{-1}$.

It is a common wisdom that DNA can be treated as an one-dimensional linear chain of stacked base pairs. We believe, that in the ground state every base pair contains bound electrons only. Then the charge is carried through DNA by excited electrons (or holes) which can jump between the base pairs. Below we propose a simple model which, to our meaning, reflects basic features of the electron transport in DNA. The picture includes the following ingredients:

(i) The excited electron states at the base pairs are separated by energy spacing larger than temperature and therefore thermally are practically not excited;

(ii) Thermal motions of the DNA base pairs are elastic vibrations with a characteristic frequency $\omega_{b}$;

(iii) Efficient charge transfer between neighboring base pairs takes place for rare events;

(iv) The Coulomb interactions between the electrons and holes can be neglected for the description of hopping transport.

Let us explain the point (iii) in more detail. For the static equilibrium DNA helix charge hopping is expected to be negligibly small since there is no significant electronic overlapping between adjacent base pairs. Nevertheless, sometimes, due to thermal fluctuations, exclusively favourable for hopping configurations of the base pairs occur, when an efficient hopping is possible. If the separation between the pairs is larger than the amplitude of their thermal vibrations, then probability of such events (which can be called "contacts") is small. Duration of the contact can be estimated as the characteristic oscillation time $\omega_{b}^{-1}$.

Now we discuss a correspondence of the assumptions enlisted above and experimental data. As a guide line we 
use not only data known from the DNA literature but as well the data obtained for a wide range of organic linear chain polymers of stacked planar molecules (for a review see 24). The contacts are related to mutual displacements and orientations of adjacent base pairs. Probably, hopping matrix elements are mostly sensitive to the relative rotations (twist fluctuations) of the base pairs (see, e.g., [25]). The characteristic frequency of these fluctuations, $\omega_{b}$, is usually estimated as being in the region $10^{11} \div 10^{12} s^{-1}$. A small probability of the contacts is confirmed by experiment showing that the characteristic electronic hopping time $\tau$ is larger than $\omega_{b}^{-1}$, in the experiments 88,10,17,18 $\omega_{b} \tau=10^{2} \div 10^{3}$. Our first assumption (i) requires $\Delta E>T$, where $\Delta E$ is the spacing in the spectrum of electron excitations for a base pair. The magnitude of $\Delta E$ can be measured directly, for the experiments 823 $\Delta E>500 K$. Therefore the inequality is satisfied. Rough macroscopical estimations of the Coulomb interaction $U_{c}$, as well as ab initio molecular orbital calculations of $U_{c}$, give few $m e V$ [26], and thus Coulomb energy appears to be smaller (though of the same order) than $\hbar \omega_{b}$. We believe that it is enough to justify neglecting Coulomb interaction.

The above reasoning leads to an $1 d$ hopping Hamiltonian for the electrons

$$
\mathcal{H}=\hbar \sum_{i}\left(\xi_{i} a_{i}^{+} a_{i+1}+\xi_{i}^{*} a_{i+1}^{+} a_{i}\right)
$$

Here $a_{i}$ and $a_{i}^{+}$are electronic annihilation and creation operators at the site (i.e. the base pair) with the number $i$, and $\xi_{i}$ are the hopping amplitudes, which are timedependent quantities. The equations for the Heisenberg operators $a_{i}$ are

$$
\partial_{t} a_{i}=-i \xi_{i} a_{i+1}-i \xi_{i-1}^{*} a_{i-1} .
$$

We assume that different $\xi_{i}$ possess independent statistics, since $\xi_{i}$ are related to independent thermal pair base fluctuations. The hopping matrix element $\xi_{i}$ can be decomposed into a constant part $\left\langle\xi_{i}\right\rangle$, that describes the coherent charge carrier motion in a completely rigid lattice, and a fluctuating part. Since the probability to jump is appreciable during rare events, the coherent part of $\xi$ can be neglected in comparison with its fluctuating part.

Note, that theoretical models based on hopping Hamiltonians similar to Eq. (1) are widely used to describe charge transport in solid state physics (see, e.g., 27,28]). For most of problems in this case the description corresponds to electron migration in a steady energy landscape, including thermally activated jumps over barriers and quantum tunneling through the barriers. It is quite different from our case.

We assume that DNA molecules can be treated as homogeneous ones. Though the molecules are constructed from four different nitrous bases, experimental data $\$ 822]$, as well as numerical first principle calculations [26, 29, 30] show, that the sequence of base pairs is not a decisive factor which determines electron transport in DNA. Quantitatively this condition can be formulated as $\delta E<\hbar \omega_{b}$, where $\delta E$ is an energy spacing between the (lowest excited) electron energy levels at different base pairs. The values of $\delta E$, known mainly from numerical electronic structure calculations [26, 29,30], are of order of $m e V$. Thus $\delta E$ is smaller than $\hbar \omega_{b}$ for $\omega_{b}$ given above, that justifies the picture. Besides some experiments (see, e.g., 23]) are performed for artificial homogeneous DNA, where $\delta E=0$. One expects that, due to the hopping, electron diffusion occurs on large time scales. For $\xi$, treated as a white noise, it was demonstrated in the papers [31,32. Though our case is essentially different, there is good reason to believe, that the same behavior should be observed on time scales larger than the hopping time $\tau$.

Below, we examine the particular case related to the fluorescence measurements, reported in the papers [8 23]. The donors are photo-excited and effects, related to the excited electron motion to the acceptors, are monitored. The energetic gaps $\delta E_{d}$ and $\delta E_{a}$ between the donor and the acceptor and the base pairs between them, are crucial for the hopping rate. The values of $\delta E_{d}$ and $\delta E_{a}$ (known mainly from ab initio numerical calculations [15, 26, 29, 30]) can be estimated as $10^{2} \mathrm{meV}$. We see, that the inequalities $\delta E_{d}, \delta E_{a} \gg \hbar \omega_{b}$ are satisfied. The electron is always bound to the acceptor site more strongly than to a standard base pair, that is $\delta E_{a}>0$. As to the donors, the sign of $\delta E_{d}$ can be either positive or negative. If $\delta E_{d}$ is negative then a scheme of the electronic charge transfer from the donor to the acceptor is quite simple. Initially, the electron leaves the donor, jumping to the neighbor site, and then jumps between the standard base pairs, trapping finally at the acceptor. The case $\delta E_{d}>0$ is more complicated. In order to have a driving force for the donor-acceptor charge transfer process the final state with the charge bound to the acceptor should be energetically favourable, that is the inequality $\delta E_{a}>\delta E_{d}$ has to be satisfied. However, there are some base pairs inbetween which play the role of the potential barrier for the electron. Therefore there are two possibilities for the electron to come to the acceptor. The first possibility is to jump initially from the donor to the neighbor site and then to move to the acceptor due to multistep hopping over the standard base pairs. The second possibility is the unistep (direct) quantum tunneling from the donor to the acceptor through the barrier.

Since $\delta E_{d} \gg \hbar \omega_{b}$, the probability for the electron to jump from the donor to the neighboring base pair due to dynamics of $\xi$ is negligible. At $\delta E_{d}>0$ such a jump is possible if the electron absorbs a high-frequency phonon with the frequency $\omega_{p h} \sim \omega_{d}\left(=\delta E_{d} / \hbar\right)$. Correspondingly, at $\delta E_{d}<0$ the electron jump from the donor is accompanied by emitting high frequency phonons. Such 
dynamical vibrations with periods as short as tens femtoseconds (i.e. phonons with $\omega_{p h} \sim 10^{14} s^{-1}$ ) were reported in the literature [33 35]. Since $\hbar \omega_{p h}>T$, occupation numbers of such phonons are small. Thus, for $\delta E_{d}>0$ the probability for the electron to jump from the donor to the neighboring site contains two small factors: the probability of the contact and the probability to absorb the high frequency phonon. It corresponds to the experimental situation where only a small fraction of the electrons are transported from the donor to the acceptor.

When the electron leaves the donor, it starts to jump between the donor and the acceptor. It can return to the donor or can come to the acceptor. If $\delta E_{d}<0$ then the probability to return to the donor is negligible. We assume that even at $\delta E_{d}>0$ the probability of the electron to jump to the donor or to the acceptor is smaller than the probability to jump to the standard base pair. There are two reasons for the assumption. First, the donors and the acceptors have chemical structures, different from the standard base pairs, that hinders for the contacts. Second, the jump has to be accompanied by the phonon emission, that diminishes its probability. The same is valid for the acceptor. Thus before being finally trapped at the acceptor site, the electron jumps many times back and forth over the base pairs between the donor and the acceptor, "smearing out" homogeneously over the all intermediate base pairs. Then the relative probability for the electron to come to the acceptor is determined by the ratio of the probabilities for the electron to jump to the donor and to the acceptor from adjacent base pairs. This relative probability appears to be independent of the separation $x$ between the donor and the acceptor. That explains why the rate of charge transfer sometimes is almost insensitive to the relative loading of donors and acceptors (see, e.g., 36, 37]). The above picture implies, that the total donor-acceptor charge transfer time should be larger than the electronic hopping time $\tau$, and it conforms to experimental data (see, e.g., [8]).

Now we consider the quantum tunneling for the electron, strongly attached to the donor, that is the case $\delta E_{d}>0$. Though the potential barrier depends on time, at the condition $\delta E_{d} \gg \hbar \omega_{b}$ the probability for the electron tunneling from the donor to the acceptor can be calculated in the adiabatic approximation. To examine the tunneling process, one should consider the quasistationary electron state bound at the donor. In the spirit of our picture we assume $\xi \ll \omega_{d}$. Then the energy of the bound state is close to $-\delta E_{d}$. Substituting $\partial_{t}$ by $i \omega_{d}$ in Eq. (2), one obtains for the state

$a_{i}=-\frac{\xi_{i-1}^{*}}{\omega_{d}} a_{i-1}$ if $i>0, \quad a_{i}=-\frac{\xi_{i}}{\omega_{d}} a_{i+1}$ if $i<0$,

where we used the condition $\xi \ll \omega_{d}$. Then the probability for the electron to be at the site $n$ is determined by the average

$$
\left\langle a_{n}^{+} a_{n}\right\rangle=\omega_{d}^{-2 n}\left\langle\left|\prod_{i=1}^{n} \xi_{i-1}\right|^{2}\right\rangle .
$$

Quantum averaging and averaging over statistics of $\xi$ are performed at deriving Eq. (4). Besides at the derivation we substituted $\left\langle a_{0}^{+} a_{0}\right\rangle \approx 1$ justified by $\left\langle a_{n}^{+} a_{n}\right\rangle \ll 1$. Note that the probability (四) is determined by the simultaneous statistics of $\xi$. Remind that different $\xi_{j}$ are assumed to be statistically independent. Therefore the average in the right-hand side of Eq. (4) is a product of $\left\langle\left|\xi_{j}\right|^{2}\right\rangle$. For the standard pairs the quantities can be regarded as site independent ones. Therefore the probability of the electron to be at the nearest to the acceptor site is proportional to $\left\langle|\xi|^{2}\right\rangle^{n} / \omega_{d}^{2 n}$ where $n$ is the number of the standard pairs between the donor and the acceptor.

The jump of the electron from the bound state to the acceptor is accompanied by the phonon emission. However, the only $x$-dependent factor in the probability of the process is related to the average charge occupation number of a site $n$ near the acceptor, established above. Thus, we obtain for the probability the exponential law $\exp (-\beta x)$ with

$$
\beta=a^{-1} \ln \left(\omega_{d}^{2} /\left\langle|\xi|^{2}\right\rangle\right),
$$

Here $\xi$ is the hopping probability for the standard base pairs and $a=3.4 A$ is the distance between the base pairs in DNA. Note, that $\omega_{d}$ depends on the donor type, whereas the average $\left\langle|\xi|^{2}\right\rangle$ is mainly related to base pair vibrations. It follows from the above consideration, that the exponential law implies the condition $\beta>a^{-1}$. This conclusion is in agreement with the majority of published experimental data. Reported in [21] the value $\beta=0.1 \AA^{-1}$ (thus smaller than $a^{-1}$ ) is, probably, related to an attempt to fit a complex behavior (including two processes: diffusion and tunneling) by a simple exponential law.

Let us stress that the quantum tunneling analyzed above is not a standard (static) tunneling described in textbooks. We have deal with dynamic tunneling which can be effective only when due to fluctuations of $\xi$ there occurs some kind of a "bridge" from the donor to the acceptor. The exponential law, we found, is explained in fact by a small probability to have such a bridge, which is realized, when simultaneously many contacts between base pairs occur. In addition, the probability of this kind of tunneling is strongly dependent on the temperature via $\left\langle|\xi|^{2}\right\rangle$. It is natural to assume that $\xi$ exponentially depends on the relative displacement $u$ of the neighboring base pairs. Then (in the harmonic approximation) $\ln \left\langle|\xi|^{2}\right\rangle$ contains the term, proportional to $\left\langle u^{2}\right\rangle$, which is proportional to the temperature $T$. Thus we arrive at the expression

$$
\beta a=c_{1}-c_{2} T,
$$


where $c_{1}$ and $c_{2}$ are temperature-independent factors. They can be extracted from the paper [20]: $c_{1} \approx 4$, $c_{2} \approx 0.01 K^{-1}$. The values are in agreement with rough estimates $c_{1} \sim a / b, c_{2} \sim k_{B} /\left(M \omega_{b}^{2} b^{2}\right)$, where $k_{B}$ is the Boltzmann constant, $M$ is the base pair mass, and $b$ is an electronic penetration length. It can be estimated as $b \sim \hbar / \sqrt{m E} \sim 1 \AA$, where $m$ is is the electron effective mass and $E$ is its binding energy at the base pair.

To conclude, for the electron, strongly bound to the donor, we established two different charge transfer mechanisms: diffusion and tunneling. The diffusion leads to the charge transfer probability independent of the donoracceptor distance $x$. However, the probability contains the small factor related to the electron jump from the donor to a neighboring site. The tunneling leads to the exponential dependence of the probability on $x$ (with the temperature-dependent length $\beta^{-1}$ ). Therefore, it is not efficient for large distances. Thus, the exponential law has to be observed for small distances $x$ whereas for large distances the charge transfer rate has to be independent of the donor-acceptor distance. Just this kind of behaviour was reported very recently [23]. For the case of the electron weakly bound to the donor the hopping should always dominate over quantum tunneling. That explains why the rate of charge transfer sometimes does not behave exponentially even for small $x$ [36, 37.

Note that in some cases the interaction of light, ionizing radiation or chemically active reagents with DNA can result in loss of an electron at a specific site with formation of a hole. In this case the charge transport through DNA can be provided by holes (see, e.g., |14). The key issues for positive charge carrier transport are the same as for the electrons. As far as the physical picture of charge transport is essentially the same for both kind of carriers, it can be described in the framework of the same approach.

The research presented in this publication was made possible in part by RFFR Grant 00-02-17785. Fruitful discussions with A. Iosselevitch, Yu. Evdokimov, V. Golo, D. Bicout, and T. Costi are gratefully acknowledged.

[1] S. D. Eley and D. L. Spivey, Trans. Faraday Soc., 58, 411 (1962).

[2] J. Ladik, Acta Phys. Acad. Sci. Hung. 11, 239 (1960).

[3] H. W. Fink and C. Schonenberg, Nature, 398, 407 (1999).

[4] A. Yu. Kasumov et al., Science, 291, 280 (2001).
[5] Y. Okahata, T. Kobayashi, K. Tanaka, and M. Shimomura, J. Am. Chem. Soc., 120, 6165 (1998).

[6] P. Tran, B. Alavi, and G. Gruner, Phys. Rev. Lett., 85, 1564 (2000).

[7] D. Porath, A. Bezryadin, S. de Vries, and C. Dekker, Nature, 403, 635 (2000).

[8] M. R. Arkin et al., Science, 273, 475 (1996).

[9] F. D. Lewis et al., Science, 277, 673 (1997).

[10] P. F. Barbara and E. J. C. Olson, Advances in Chemical Physics, 107, Ch. 13, Wiley, 1999.

[11] E. Meggers, M. E. Michel-Beyerle, and B.Giese, J. Am. Chem. Soc., 120, 12950 (1998).

[12] D. N. Bertrand, S. Priyadarshy, and S. M. Risser, Chem. Biol., 4, 3 (1997).

[13] C. Wan, et al., Proc. Natl. Acad. Sci. USA, 96, 6014 (1999).

[14] P. T. Henderson, D. Jones, G. Hampikian, Y. Kan, and B. G. Shuster, Proc. Natl. Acad. Sci. USA, 96, 8353 (1999).

[15] J. Jortner, M. Bixon, T. Langenbacher, M. E. MichelBeyerle, Proc. Natl. Acad. Sci. USA, 95, 12759 (1998).

[16] F. C. Grozema, Y. A. Berlin, and L. D. A. Siebels, Int. J. Quant. Chem., 75, 1009 (1999).

[17] D. B. Hall, R. E. Holmkin, J. K. Barton, Nature, 382, 731 (1996).

[18] D. B. Hall, J. K. Barton, J. Am. Chem. Soc., 119, 5045 (1997).

[19] M. R. Arkin, E. D. A. Stemp, S. C. Pulver, and J. K. Barton, Chem. Biol., 4, 369 (1997).

[20] A. K. Felts, W. T. Pollard, and R. A. Friesner, J. Phys. Chem., 99, 2929 (1995).

[21] K. Fukui, K. Tanaka, Angew. Chem. Int. Ed., 37, 158 (1998).

[22] C. Wan, T. Fiebeg, O. Schiemann, J. K. Barton, and A. H. Zewail, Proc. Nat. Acad. Sci. USA, 97, 14052 (2000).

[23] B. Giese, J. Amaudrut, et al., Science, 412, 318 (2001).

[24] D. Jerome and H. Schultz, Adv. Phys., 31, 299 (1982).

[25] R. Bruinsma, G. Gruner, M. R. D'Orsogona, J. Rudnik, Phys. Rev. Lett., 85, 4393 (2000).

[26] L. Ducasse, A. Fritsch, F. Castet, J. de Phys. IV, 10, 3 (2000).

[27] J. Callaway, Quantum Theory of the Solid State (Acad. Press, New York, 1974).

[28] J. Appel, in Solid State Physics, 21, (editors F. Seits, D. Turnbull, and H. Ehrenreich) 193 (1968).

[29] P. J. de Pablo, et al., Phys. Rev. Lett., 85, 4992 (2000).

[30] J. J. Ladik, Phys. Repts., 313, 171 (1999).

[31] H. Haken and P. Reineker, Z. Phys., 249, 253 (1972).

[32] H. Haken and G. Strobl, Z. Phys., 262, 135 (1973).

[33] M. B. Hakim, S. M. Lindsay, and J. Powel, Biopolymers, 24, 1185 (1984).

[34] E. B. Brauns, C. J. Murphy, and M. A. Berg, J. Am. Chem. Soc., 120, 2449 (1998).

[35] N. V. Hud, M. Polak, Curr. Opin. Struct. Biol., 11, 292 (2001).

[36] C. J. Murphy, et al., Science, 262, 1025 (1993).

[37] J. W. Evensoon, M. Karplus, Science, 262, 1247 (1993). 